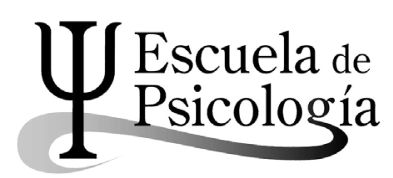

Wímb lu, Rev. electrónica de estudiantes Esc. de psicología, Univ. de Costa Rica. 10 (2): 55-65, 2015 / ISSN: 1659-2107

\title{
ADAPTACIÓN Y ESTUDIO DESCRIPTIVO DEL EXPERIMENTO SIMON EN UNA MUESTRA DE ESTUDIANTES ECUATORIANOS
}

\author{
Adaptation and descriptive study of Simon experiment \\ in a sample of ecuadorian students
}
Carlos Alberto Ramos Galarza ${ }^{a}$
María Eulalia Guevara Vega ${ }^{b}$ Mónica Bolaños Pasquel ${ }^{c}$ David Ramos Galarzad

\begin{abstract}
Resumen: Introducción. El experimento Simon es una tarea que permite evaluar el control inhibitorio y tiempo de reacción ante un estímulo que genera una tendencia automática de respuesta en el ser humano. Actualmente no existe investigación alguna que haya buscado adaptar este experimento en el contexto ecuatoriano. Objetivo. Adaptar y realizar un estudio descriptivo y correlacional del experimento Simon en una muestra de estudiantes ecuatorianos. Materiales y Método. La muestra estuvo conformada por 81 participantes entre 6 y 15 años de edad. Dentro del análisis se utilizó estadística descriptiva y paramétrica. Resultados. Se encontró que el tiempo de reacción es menor a medida que la edad avanza. Además, se observó que existe una tendencia de mejora del control inhibitorio desde los seis a los doce años, edad en donde existe una disminución en su maduración, la cual vuelve a mejorar alrededor de los quince años de edad. Conclusión. El experimento Simon presenta las características necesarias para ser aplicado en estudiantes ecuatorianos. Esta investigación nos proyecta a realizar nuevos estudios en donde podamos determinar los parámetros normativos de las respuestas en inhibición y tiempo de reacción de estudiantes a nivel nacional, tanto en población clínica como con desarrollo regular.
\end{abstract}

Palabras Clave: Control inhibitorio, Experimento Simon, Psicología Experimental, Tiempo de reacción.

a Universidad de las Américas. Neuropsicólogo Clínico. Docente Escuela de Psicología. Ecuador. Correo: ps_carlosramos@hotmail.com.

b Universidad Andrés Bello. Neurorehabilitadora. Chile. Correo: ma.eulaliaguevara@gmail.com.

c Centro de Investigación, Diagnóstico y Rehabilitación en Neuropsicología. Psicóloga. Ecuador. Correo: monicabolanos@gmail.com.

d Universidad Tecnológica Israel. Investigador Educativo. Ecuador. Correo: dr_david_ramos@hotmail.com.

Recepción: 23/6/2015 Aceptación: 17/7/2015. 


\begin{abstract}
Introduction. The Simon experiment is a task for assessing the inhibitory control and reaction time. Currently there is no research that has sought to adapt this experiment in the Ecuadorian context. Aim. To adapt the Simon experiment in a sample of Simon Ecuadorian students. Materials and Methods. The sample consisted of 81 participants between 6 and 15 years old. In the analysis we used descriptive and parametric statistical process. Results. It was found that the reaction time decreases as you age. It was also noted that there is a trend of improvement of inhibitory control from six to twelve years of age, age where there is a decrease in its maturation, which improves again about fifteen years old. Conclusion. Simon experiment presents the characteristics needed for being applied in Ecuadorian students. In future research we aim to determine the normative parameters of inhibition errors and reaction time for ecuadorian students.
\end{abstract}

Key Words: Experimental Psychology, inhibitory control, reaction time, Simon Experiment.

\title{
Introducción
}

El experimento basado en el efecto Simon es una tarea computarizada que permite evaluar la función ejecutiva control inhibitorio (Romero-Ayuso, Maestú, González-Marqués, Romo-Barrientos y Andrade, 2006). Kunde y Stocker (2002) describen que el objetivo de esta tarea es la de inhibir la tendencia de una respuesta automática. Barkley (2003) señala que el ser humano, en este experimento, presenta una predisposición para responder hacia el hemicampo en el cual se presenta un determinado estímulo, situación que debe ser inhibida.

Simon y Rudell en 1967 desarrollaron este experimento, al cual se lo ha denominado Simon por su creador principal, en éste se presentan estímulos en los hemicampos izquierdo y derecho, a los cuales los participantes deben contestar aplastando una tecla con su dedo índice izquierdo o derecho. Según Del Valle-Inclán, Rumbo, Redondo y Lamas (1995,pp. 139-140) en un experimento Simon clásico "los estímulos tienen un atributo relevante (color, forma, etc.) y un atributo irrelevante (localización espacial)". En el caso del experimento Simon de interés del presente estudio es el desarrollado por el Sistema de Construcción de Experimentos en Psicología PEBL (Mueller, 2015), el cual contiene dos estímulos: un círculo azul y un círculo rojo. Ante el primer estímulo se debe presionar un botón con la mano derecha y ante el segundo, el botón de la mano izquierda. El atributo irrelevante del experimento es la posición espacial en la cual se presenta el estímulo, es decir, el círculo azul puede presentarse en el hemicampo derecho o izquierdo y ante su presentación siempre se debe responder con el botón derecho, lo cual genera el efecto de interferencia a inhibir (Kornblum, Hasbroucq y Osman, 1990). Además, el experimento Simon permite medir el tiempo de reacción, según Londoño (2009, pp. 98-99) "esta evaluación permite medir la demora más leve entre una respuesta habitualmente voluntaria y una incitación inicial o estímulo dado", mediante este experimento es interesante poder valorar el tiempo en milisegundos, en el cual un participante demora en responder ante un estímulo, luego de que éste es presentado. 
El experimento Simon desarrollado por el sistema PEBL (Mueller y Piper, 2014) ha sido creado para utilizarse en países de habla inglesa o portugués, sin embargo, no se cuenta con una versión adaptada al español o contextualizada en algún país de habla hispana.

Dentro del contexto descrito nos proponemos como objetivo el adaptar el experimento Simon PEBL a un contexto latinoamericano, específicamente en Ecuador, y realizar un estudio descriptivo de las medidas obtenidas en este experimento en una muestra de estudiantes.

\section{Materiales y métodos}

\section{Participantes}

La muestra estuvo conformada por 81 estudiantes entre 6 y 15 años de edad, pertenecientes al sistema público educativo de Ecuador, 47 hombres (58\%) y 34 mujeres (42\%), la media de edad de los hombres es 9.85 años (DE 2,73), en las mujeres la media es 10,41 años (DE 2,24). El nivel educativo de los y las participantes va desde tercero de educación básica hasta primero de bachillerato.

\section{Diseño de la investigación}

El enfoque es cuantitativo, el diseño y alcance del estudio es descriptivo y correlacional, con una temporalidad de tipo transeccional (Hernández, Fernández y Baptista, 2010).

\section{Instrumentos}

Experimento basado en el efecto Simon construido en el sistema PEBL (Mueller, 2015). Consiste en una tarea cognitiva que evalúa la inhibición de una tendencia de respuesta (Kunde y Stocker, 2002). En la tarea se presentan un círculo de color azul (a responder con la mano derecha) o rojo (a responder con la mano izquierda). Para el ingreso de las respuestas adaptamos una caja de botones que sustituyeron las teclas Shift izquierda y derecha con las que normalmente se responde en el sistema PEBL. Entre las presentaciones de los estímulos existe una cruz en el centro de la pantalla, la cual desaparece cuando se presenta uno de los círculos. Los círculos se pueden presentar dentro de cualquier campo visual, por ejemplo, el azul puede presentarse en el hemicampo izquierdo y el participante debe responder con su mano derecha, debiendo activar su control inhibitorio para no actuar de forma automática hacia el hemicampo en el cual se presenta el estímulo (Barkley, 2003). El número de ensayos es 140, los cuales son presentados de forma aleatoria en cada experimento, el tiempo que toma la 
realización del experimento es alrededor de 6 minutos (en la figura 1 se presenta una captura de pantalla de éste).

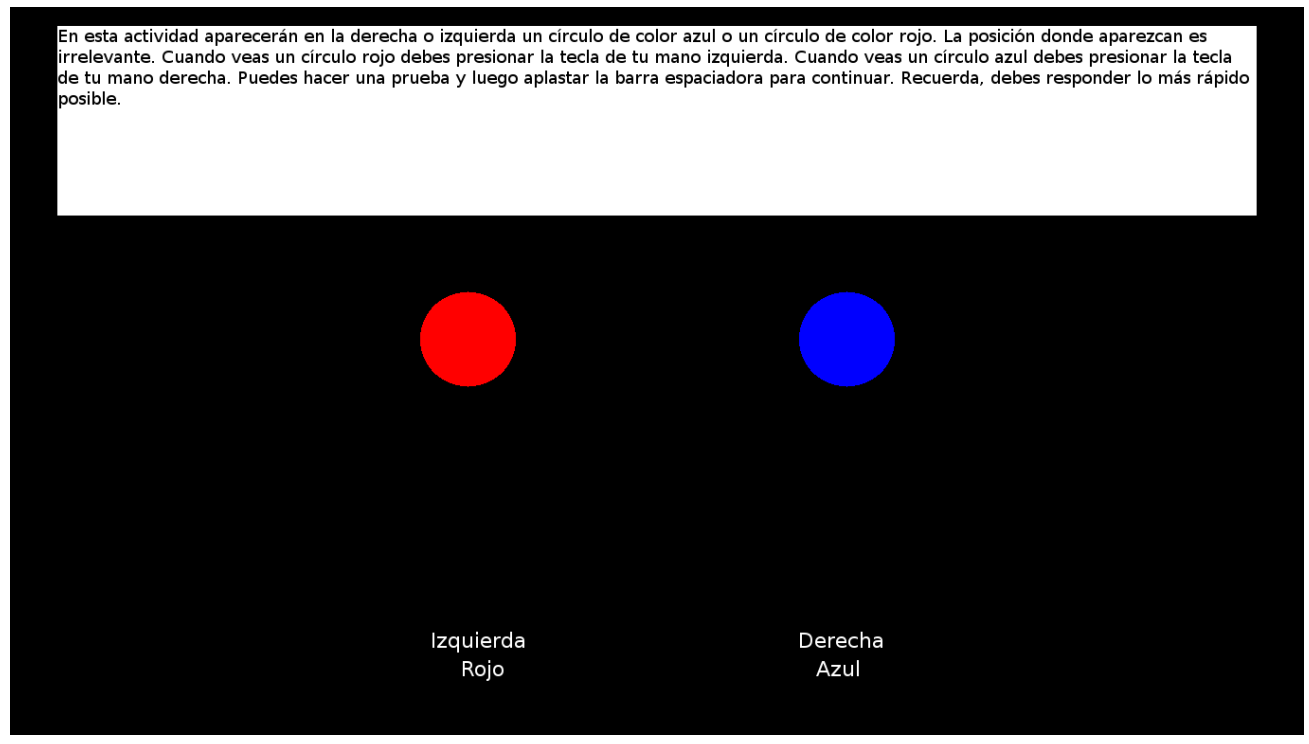

FIGURA 1.

Captura de pantalla del experimento Simon.

\section{Análisis de datos}

Con la finalidad de describir los valores obtenidos por los participantes, según el género y grupo etario, se utilizó estadística descriptiva (medidas de tendencia central y dispersión). Para analizar si se presentan diferencias en las puntuaciones obtenidas en el experimento, según el género y grupo etario, se utilizaron las técnicas paramétricas $T$ de Student y ANOVA de una vía. Con la finalidad de correlacionar la variable edad con el tiempo de reacción y errores de inhibición se utilizó el proceso no paramétrico de correlación de Spearman. Todos los análisis se los realizó en el software estadístico SPSS versión 20 (IBM, 2011).

\section{Procedimiento}

En primer lugar se procedió a la traducción de las instrucciones del experimento. Para cumplir con este fin se reconfiguró el código fuente del sistema PEBL, en el cual se adaptó las instrucciones del experimento al contexto lingüístico ecuatoriano. Una vez logrado este punto se solicitó el juicio experto de tres profesionales con grado de maestría en neuropsicología y experiencia en la evaluación de las funciones ejecutivas. Luego de realizadas las modificaciones del texto y características del experimento, en relación a las sugerencias recibidas, se procedió a realizar un estudio piloto con 5 estudiantes, que no formaron parte 
del estudio, dentro del mismo grupo etario y características de los participantes. Una vez que se contó con el experimento en las condiciones idóneas, la siguiente fase del estudio consistió en solicitar autorización a las autoridades educativas. Posteriormente se envió a los estudiantes seleccionados el consentimiento informado y se entregó el asentimiento de participación a cada participante. Una vez que se contó con las firmas de los documentos descritos se procedió a ejecutar las evaluaciones de los participantes. Cada evaluación fue realizada en un contexto sin distracciones y en todo momento del estudio se respetó los parámetros éticos para la investigación en los seres humanos declarados en Helsinki (Williams, 2008).

\section{Resultados}

En la media de errores en los ensayos incongruentes en donde se debe activar el control inhibitorio, los participantes de género masculino presentan una media de 15,85 errores (DE 8,30), mientras que las mujeres presentan una media de errores de 13 errores (DE 7,45). En la figura 2 se presenta de forma gráfica estos resultados.

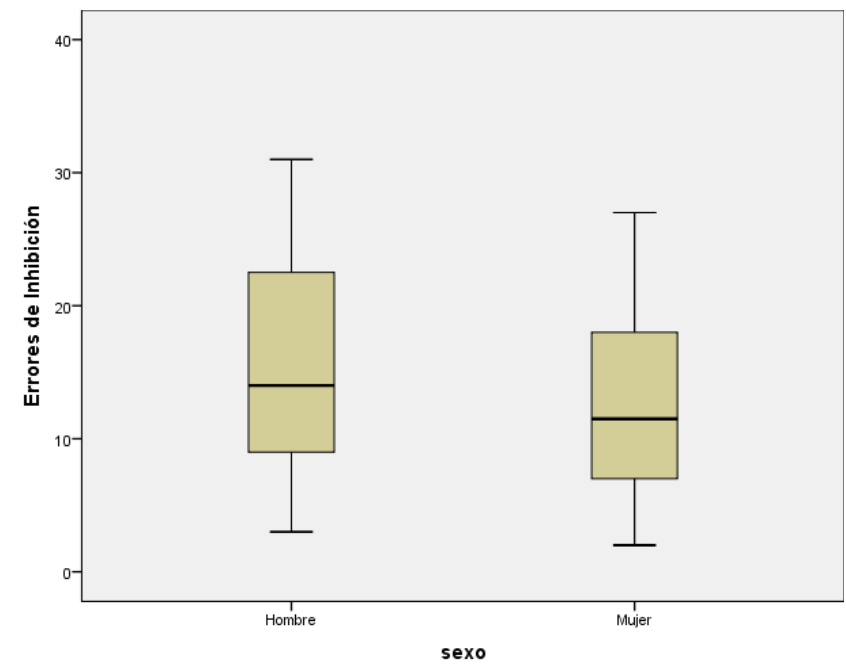

FIGURA 2.

Descripción gráfica de los errores de inhibición cometidos por los participantes en la investigación.

En relación a los puntajes obtenidos en la medida tiempo de reacción, los hombres presentan una media de 806,73 milisegundos (DE 179,56), mientras que las mujeres reportan una media de 810,68 milisegundos (DE 176,23). En la figura 3 se presenta de forma gráfica dichos resultados. 


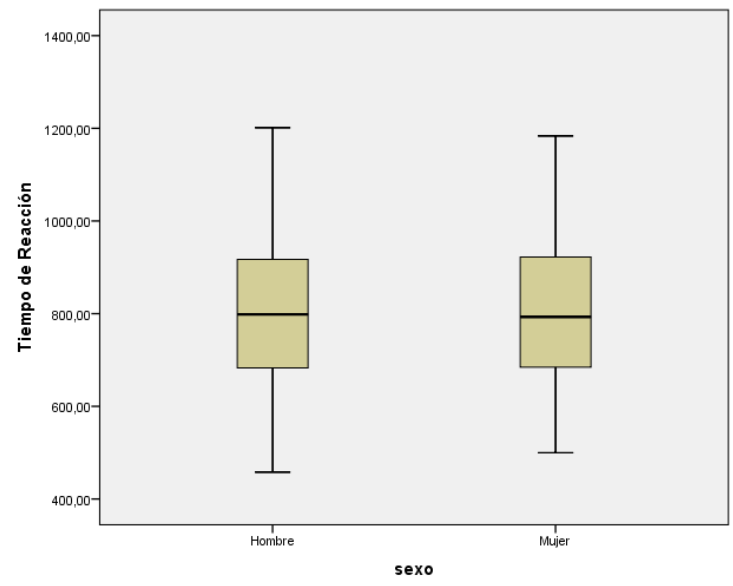

FIGURA 3.

Descripción gráfica del tiempo de reacción obtenido por los participantes de género masculino y femenino.

En cuanto a la cantidad de errores de inhibición según el grupo etario al que pertenecen los participantes, se observa que a la edad de seis años la media de errores es 21,5 (DE 7,78), siete años 21,85 (DE 6,48), ocho años 15,33 (DE 6,99), nueve años 10 (DE 4,63), diez años 14 (DE 7,10), once años 10 (DE 5,92), doce años 7,11 (DE 4,19), trece años 14,33 (DE 7,20), catorce años $15,20(10,89)$ y a los quince años de edad la media de errores es 18,40 (DE 10,69). Como complemento de los datos indicados, en la figura 4 se presenta de forma gráfica los errores de inhibición según grupo etario y género de los participantes.

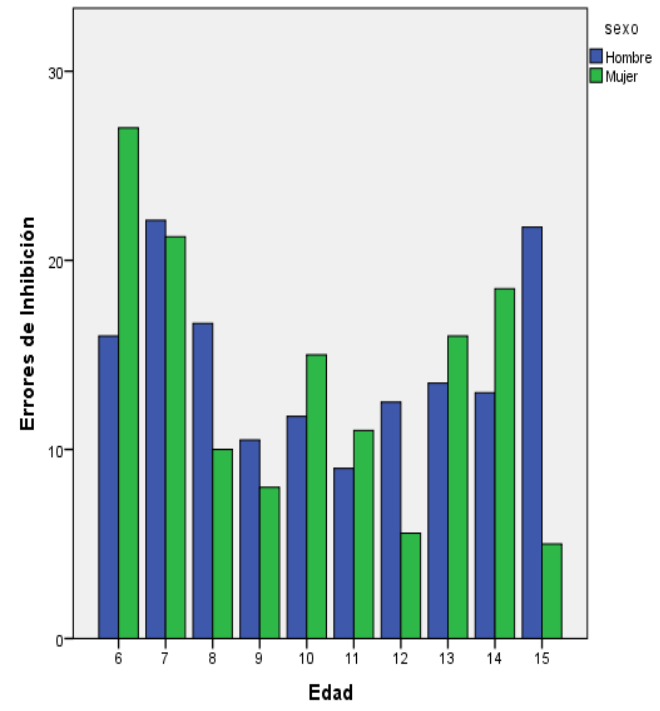

FIGURA 4.

Representación gráfica de los errores de inhibición cometidos por los y las participantes según el grupo etario al que pertenecen. 
En el tiempo de reacción de los participantes, según el grupo etario, se observa que a la edad de seis años la media de milisegundos de reacción entre la presentación de los estímulos y la respuesta es de 1120,41 (DE 8,05), siete años 830,65 (DE 225,88), ocho años 928,49 (DE 142,69), nueve años 851,71 (DE 90,93), diez años 799,57 (DE 130,38), once años 755,26 (DE 151,23), doce años 747,16 (DE 100,90), trece años 771,59 (DE 178,93), catorce años 625,71 $(148,00)$ y a los quince años de edad la media de milisegundos de tiempo de reacción es 666,93 (DE 177,07). Para complementar los datos descritos, en la figura 5 se describen los resultados obtenidos por tiempo de reacción organizados en grupo etario y género.

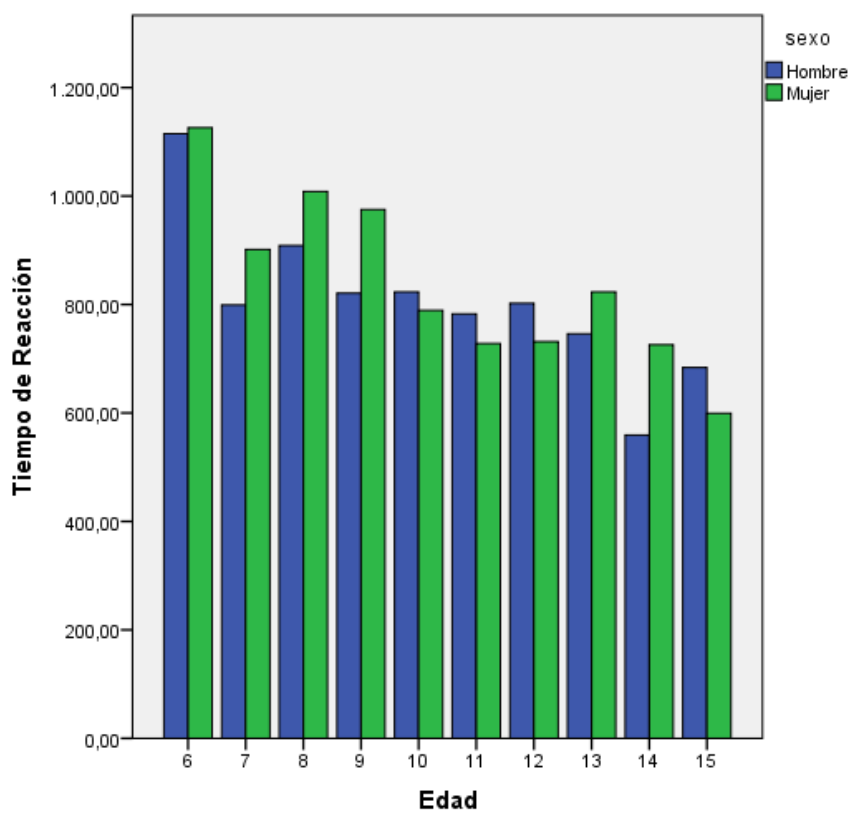

FIGURA 5.

Representación gráfica del tiempo de reacción de los y las participantes según el grupo etario al que pertenecen.

En el análisis correlacional se obtuvieron relaciones estadísticamente significativas ( $p<0,001$ a nivel bilateral) entre la variable edad con: (a) tiempo de reacción $\left(r_{s}=-0,43\right)$ y $(b)$ con el número de errores de inhibición $\left(r_{s}=-0,32\right)$.

Según el análisis estadístico $t$ se encuentra que no existen diferencias estadísticamente significativas entre hombres y mujeres en total de errores de inhibición t $(79,2)=1,59, p=0,12$ y en tiempo de reacción $t(79,2)=-0,09, p=0,92$.

En el ANOVA realizado se encontró que existen diferencias estadísticamente significativas entre el rendimiento en errores de inhibición $f(9,71)=3,69, p=0,001$ y tiempo de reacción $f(9,71)=3,42, p=0,002$ según el rango de edad al que pertenecen los participantes. 


\section{Discusión}

El realizar una adaptación de un experimento para evaluar la inhibición y el tiempo de reacción en población ecuatoriana es un importante avance en la investigación experimental de los procesos neuropsicológicos humanos.

En el análisis paramétrico se encontró que no existen diferencias estadísticamente significativas en la media de errores de inhibición y tiempo de reacción al compararlos entre hombres y mujeres.

En cambio, observamos que si existen diferencias en el tiempo de reacción y errores de inhibición en los diferentes grupos etarios participantes. Por ejemplo, las mujeres a los quince años de edad disminuyen notablemente el número de errores ante estímulos en los cuales deben inhibir una respuesta automática, además, se puede observar que existe una tendencia en este género a disminuir el tiempo de reacción ante los estímulos, lo cual significa que en su procesamiento de la información, a medida que avanza la edad, aumenta la eficacia y eficiencia en las respuestas ante situaciones en las cuales debe controlar su comportamiento.

En cambio, en los hombres se puede observar que los errores de inhibición ante estímulos en los cuales se debe presentar un control consciente del comportamiento aumenta en las edades relacionadas a los primeros años de educación formal, luego se observa que disminuyen considerablemente entre los 10 y 11 años de edad, para luego volver a incrementarse alrededor de los 13 años.

En relación al tiempo de reacción y número de errores de inhibición se observa que existe una tendencia de la muestra de estudio, y se lo representa mediante la correlación negativa obtenida, que significa que a menor edad mayor es el tiempo de reacción y número de errores, mientras que a mayor edad menor es el número de errores y tiempo de reacción ante un determinado estímulo. Lo cual tendría sentido con lo afirmado por Rosselli, Matute, \& Ardila (2010), quienes describen que a medida que el ser humano avanza en su edad las estructuras cerebrales avanzan en su madurez neuronal, lo cual se puede observar en el desempeño cognitivo en diferentes tareas que impliquen funciones cognoscitivas, como el experimento que hemos estudiado.

Anderson (2002) afirma que el control inhibitorio tiene una maduración progresiva, por ejemplo, a los doce meses de edad los menores son capaces de inhibir un determinado tipo de conducta, a los tres años de edad son capaces de inhibir comportamientos instintivos a pesar de que se puedan seguir observando errores de perseveración, a los seis años se observa un mejor desempeño en la capacidad de inhibir respuestas automáticas, después de los nueve años ya se evidencia una capacidad de monitorización y regular la respuesta, y a los once años ya son capaces de presentar un control inhibitorio adecuado, sin embargo, a partir de los doce años el ser humano entra en la etapa de la adolescencia, lo que 
generaría que el control inhibitorio entre dentro de otro proceso de maduración cognitiva (Feldman, 2007), en donde se evidenciará dificultades en la regulación de los impulsos, tal como se lo observa en la cantidad de errores de inhibición cometidos por la muestra del presente estudio.

\section{Conclusiones}

En el presente artículo hemos reportado el proceso de adaptación del experimento Simon en una muestra de estudiantes ecuatorianos. Dentro de los principales hallazgos resalta el que a medida que avanza en edad el ser humano mejora en sus procesos de control inhibitorio. Un dato interesante encontrado es que esta tendencia de maduración se la observa de forma progresiva desde los seis años de edad hasta el ingreso de la adolescencia, alrededor de los doce años, donde se observa que esta madurez inhibitoria disminuye para volver a madurar entre los quince años de edad. En cambio, en el tiempo de reacción se observa que este disminuye a medida que avanza la edad, es decir, los seres humanos a medida que maduramos somos capaces de procesar la información de manera más rápida.

Como línea de investigación futura se proyecta la necesidad de investigar la capacidad discriminativa del experimento Simon entre estudiantes con algún diagnóstico clínico o necesidad educativa especial con estudiantes con un nivel de desarrollo regular.

Además, esperamos en un futuro contar con un presupuesto mayor que nos permita realizar una investigación sobre el presente experimento que sea representativo a nivel nacional, donde podamos acceder a una muestra aleatoria de estudiantes de la costa, sierra, oriente y región insular de Ecuador, que nos permita determinar los parámetros normativos en la evaluación con el experimento Simon.

Finalmente, en el caso de que algún investigador desee acceder al experimento estudiado en el presente artículo puede descargarlo en el siguiente enlace: https://www.dropbox.com/sh/gseb4p4i99e6iwd/AABBPCGg4zsm0jIPbTd6 L2OIa?dl=0.

\section{Agradecimientos}

El investigador principal del equipo expresa su gratitud a: (1) la Secretaria de Educación Superior, Ciencia, Tecnología e Innovación (SENESCYT) del Ecuador por la beca recibida para cursar estudios de Doctorado en Psicología que financió el presente proyecto y (2) al Profesor PhD Shane Muller por su gentil respuesta ante las dudas que se presentaron en la programación del experimento. 


\section{Referencias}

Anderson, P. (2002). Assessment and development of executive function during childhood. Child Neuropsychology: A Journal on Normal and Abnormal Development in Childhood and Adolescence, 8 (2), 71-82.

Barkley, R. (2003). Issues in the diagnosis of attention-deficit/hyperactivity disorder in children. Brain and Development, 25 (2), 77-83.

Del Valle-Inclán, F., Rumbo, T., Redondo, M., \& Lamas, J. (1995). El efecto Simon estudiado con potenciales cerebrales relacionados con el movimiento. Psicothema, 7 (1), 139-146.

Feldman, R. (2007). Desarrollo Psicológico a través de la vida. México D.F.: Pearson Prentice Hall.

Hernández, R., Fernández, C., \& Baptista, P. (2010). Metodología de la Investigación. México D.F.: McGRAW-HILL / INTERAMERICANA EDITORES.

IBM. (2011). SPSS Statistics. IBM Corp.

Kornblum, S., Hasbroucq, T., \& Osman, A. (1990). Dimensional overlap: Cognitive basis for stimulus-response compatibility: A model and taxonomy. Psychological Review, 97, 253-270.

Kunde, W., \& Stocker, C. (2002). A Simon effect for stimulus-response duration. Quartely Journal of Experimental Psychology, 55, 581-592.

Londoño, L. (2009). La atención: un proceso psicológico básico. Pensando Psicología, 5 (8), 92-100.

Mueller, S. (2015). The Psychology Experiment Building Language (Version 0.14) [Software]. Disponible en http://pebl.sourceforge.net.

Mueller, S., \& Piper, B. (2014). The Psychology Experiment Building Language (PEBL) and PEBL Test Battery. Journal of Neuroscience Methods, 222, 250-259.

Romero-Ayuso, D., Maestú, F., González-Marqués, J., Romo-Barrientos, C., \& Andrade, J. (2006). Disfunción ejecutiva en el trastorno por déficit de atención con hiperactividad en la infancia. Revista de Neurología, 42 (5), 265-271.

Rosselli, M., Matute, E., \& Ardila, A. (2010). Neuropsicología del desarrollo infantil. México D.F.: El Manual Moderno. 
Simon, J., \& Rudell, A. (1967). Auditory S-R compability: The effect of an irrelevant cue on information processing. Journal of Applied Psychology, 51, 300-304.

Williams, J. (2008). Revising the declaration oh Helsinki. World Medical Journal, 54 (4), 120-122.

\section{(c) (i) ()}

Esta obra está bajo una licencia de Creative Commons Reconocimiento

- NoComercial - CompartirIgual (by-nc-sa) 